\title{
Effects of Calcitonin on Rheumatoid Arthritis and the Relation with Corticosteroids
}

\author{
Sumihisa Aida*1 and Koki Shimoji*2 \\ *1 Pain Clinic, Bentenbashi Hospital, Niigata, \\ *2 Department of Anesthesiology, Niigata University, School of Medicine, Niigata, Japan
}

(Received 20 March 1990, Accepted 23 March 1990)

\begin{abstract}
The effects of eel calcitonin (CT) on rheumatoid arthritis (RA) were studied. Ten MRC units of CT were intramuscularly administered twice a week during the initial 6 months and once a week during the following 12 months to 102 RA patients. Clinical symptoms and laboratory data were examined before and after 6,12 and 18 months of CT treatment. Grasping power, class, pain score, and morning stiffness were significantly improved by CT treatment. C-reacting protein, sialic acid, RA factor, and erythrocyte sedimentation rate were significantly decreased following the drug. Both $\alpha_{2}$ and $\gamma$ globulin levels also significantly decreased, whereas the albumin/globulin ratio was significantly elevated. Further, IgG, IgA and IgM levels significantly decreased. CT also improved the anemia associated with RA : the serum iron, red blood cell count, hematocrit and hemoglobin all significantly increased. CT and corticosteroid was treated in combination during the initial 6 months to $36 \mathrm{RA}$ patients. This combination therapy revealed that corticosteroids interfered with the beneficial effects of $\mathrm{CT}$. This interferation was seen even 12 months after the withdrawal of corticosteroid. No major complications occurred due to $\mathrm{CT}$ administration. These results suggest that $\mathrm{CT}$ affects the immunological mechanism underlying RA. Thus, CT appears to have a remission inducing effect on RA, and this effect is hampered by the concomitant use of corticosteroids.
\end{abstract}

Key Words : calcitonin, rheumatoid arthritis, remission induction, corticosteroid, immunoglobulin

\section{Introduction}

Rheu matoid arthritis (RA) is considered to be one of the most miserable diseases because it is associated with progressive generalized articular destruction and bone loss. Although various therapies for RA have been attempted, few have been found to be valuable. Calcitonin, an endocrine polypeptide from the thyroid gland, is widely known to inhibit bone resorption ${ }^{4,7)}$ and suggested to have an anti-inflammatory effect in rats with adjuvant arthritis ${ }^{1,6}$. These effects of calcitonin is considered to be beneficial for RA treatment.

Therefore, we used eel calcitonin for RA treatment, and reported an improving effect on 
Table 1 Summary of cases

\begin{tabular}{llrcccccc}
\hline \hline \multirow{2}{*}{ Group } & \multirow{2}{*}{ Sex } & \multirow{2}{*}{$\mathrm{N}$} & \multirow{2}{*}{ Age } & \multicolumn{4}{c}{ Stage (\%) } \\
\cline { 5 - 9 } & & & & & I & II & III & IV \\
\hline CT only & male & 21 & $56 \pm 3.0$ & 0.0 & 47.6 & 38.1 & 14.3 \\
& female & 81 & $53 \pm 1.4$ & 3.7 & 22.2 & 51.9 & 22.2 \\
\hline CT plus & male & 7 & $49 \pm 5.0$ & 0.0 & 14.3 & 57.1 & 28.6 \\
Corticosteroid & female & 29 & $55 \pm 2.3$ & 0.0 & 24.1 & 41.4 & 34.5 \\
\hline
\end{tabular}

Mean \pm SEM.

RA in preliminary studies ${ }^{2,3}$. It was suggested that this therapeutic effect seen with calcitonin was based neither on its anti-inflammatory action nor on the inhibition of bone resorption, but on its effects on the immunological mechanism. The present study was undertaken to obtain further informations to confirm our preliminary results.

\section{Methods}

The subjects of this study were 138 patients diagnosed as having RA according to the revised criteria of American Rheumatism Association ${ }^{5}$. None had received iron therapy, and non of them had any renal or hepatic disorders before this study. Their stage and class were determined according to the criteria of Steinbrocker et al. (Table 1) ${ }^{12)}$. Pain and morning stiffness were evaluated and scored into 4 grades of increasing intensity. Pain was classified as: I, no or slight pain ; II, moderate but tolerable pain ; III, painful and disturbed to work; IV, severe pain causing difficulty to move. Morning stiffness was classified as: I, disappearing within $15 \mathrm{~min}$; II, disappearing within $60 \mathrm{~min}$; III, disappearing within $3 \mathrm{hr}$; IV, continuing for more than $3 \mathrm{hr}$.

Laboratory data measured were as follows; C-reacting protein (CRP, immunodiffusion), sialic acid (UV rate assay), RA factor (LA method), protein fraction (electrophoresis), IgG, $\operatorname{IgA}$ and $\operatorname{IgM}$ (SRID), alkaline phosphatase (ALP) (method of Bessey and Lowry), inorganic phosphate (method of Fiske and Subbarow), serum calcium (OCPC method), and serum iron (Ferrosin method). Red blood cell count (RBC), hematocrit, and hemoglobin were analysed by means of Microcellcounter F-800 $\left(\right.$ Sysmex $\left.{ }^{\mathbb{R}}\right)$.

Ten MRC units of eel calcitonin (CT) were intramuscularly administered twice a week during the initial 6 months and once a week during the following 12 months to $102 \mathrm{RA}$ patients who had not previously received any immune therapy such as immunodepressants or immunomodulators (CT only group). Clinical symptoms and laboratory data were examined before and after 6,12 and 18 months of CT treatment.

$\mathrm{CT}$ and a corticosteroid were administered in combination to $36 \mathrm{RA}$ patients (CT plus corticosteroid group). They had already received a corticosteroid for RA therapy for at least one month before the start of this study. Each in this group had received a various oral doses of different kinds of corticosteroids, such as prednisolone, dexamethasone or betamethasone. In each case, their previous corticosteroid treatment was continued during the initial 6 months. During the next 6 months the doses of corticosteroids were gradually reduced to be discontinued, and during the final 6 months only CT was administered. The CT plus corticosteroid group also received $\mathrm{CT}$ at the same dosage and through the same rout as the $\mathrm{CT}$ only group.

The data for these two groups were compared before and after CT administration. Results were expressed as the mean and the standard 


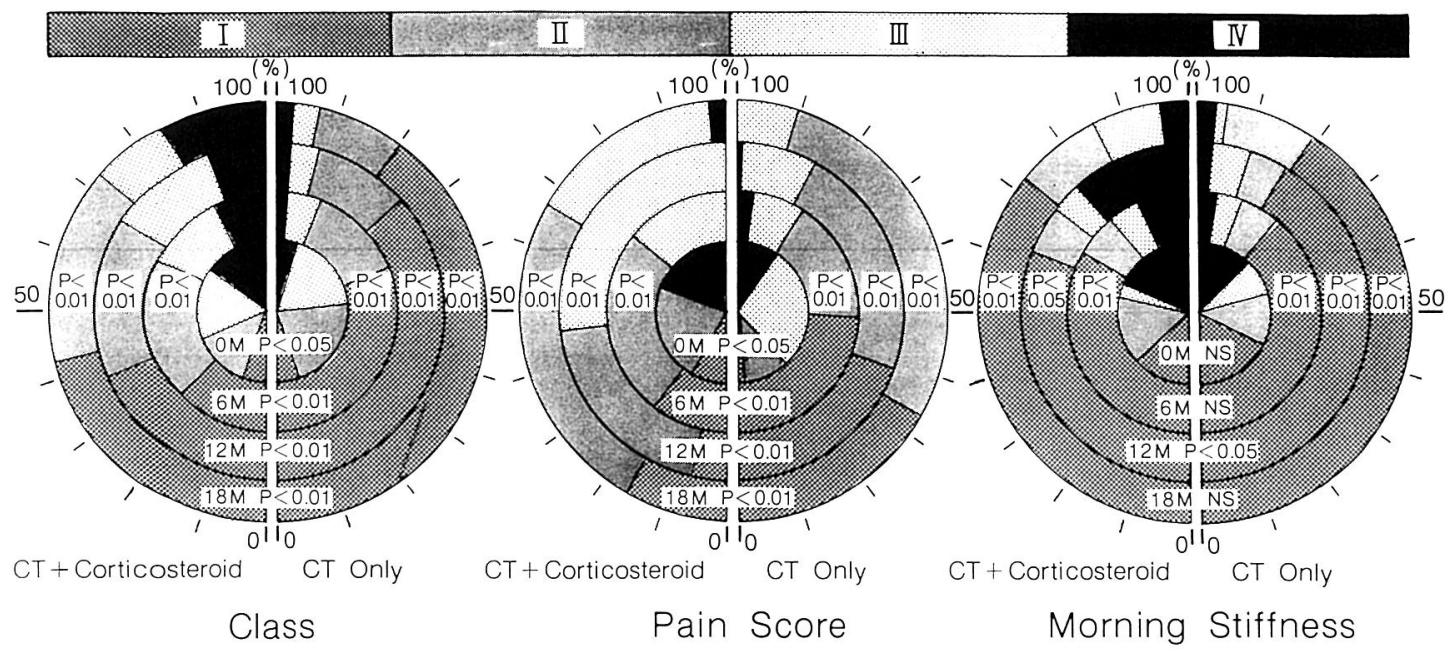

Fig. 1 Changes in class, pain score and morning stiffness of RA patients in the CT only group (right half circles) and the CT plus corticosteroid group (left half circles).

error of the mean (mean \pm SEM). Data were statistically analysed using Student's t-test or Wilcoxon's signed rank test (class, pain score, morning stiffness and RA factor), and a $\mathrm{p}$ value less than 0.05 was considered signigficant.

\section{Results}

In the CT only group, significant improvements in clinical symptoms and laboratory findings were observed after CT treatment. Significant improvements in class, pain score, morning stiffness, and grasping power (both sides) were noted (Figs. 1, 2). CRP, sialic acid, RA factor, and erythrocyte sedimentation rate (ESR) were significantly decreased (Fig. 3). Albumin/globulin $(\mathrm{A} / \mathrm{G})$ ratio was significantly elevated, while both $\alpha_{2}$ and $\gamma$ globulins significantly decreased. Significant decreases were also noted in IgG, IgA, and IgM (Fig. 4). The anemia associated with RA was improved; there were significant increases in serum iron, RBC, hemoglobin, and hematocrit (Fig. 5). ALP significantly decreased, and a slight decrease in serum calcium was observed (Fig. 6).

In the CT plus corticosteroid group, quite

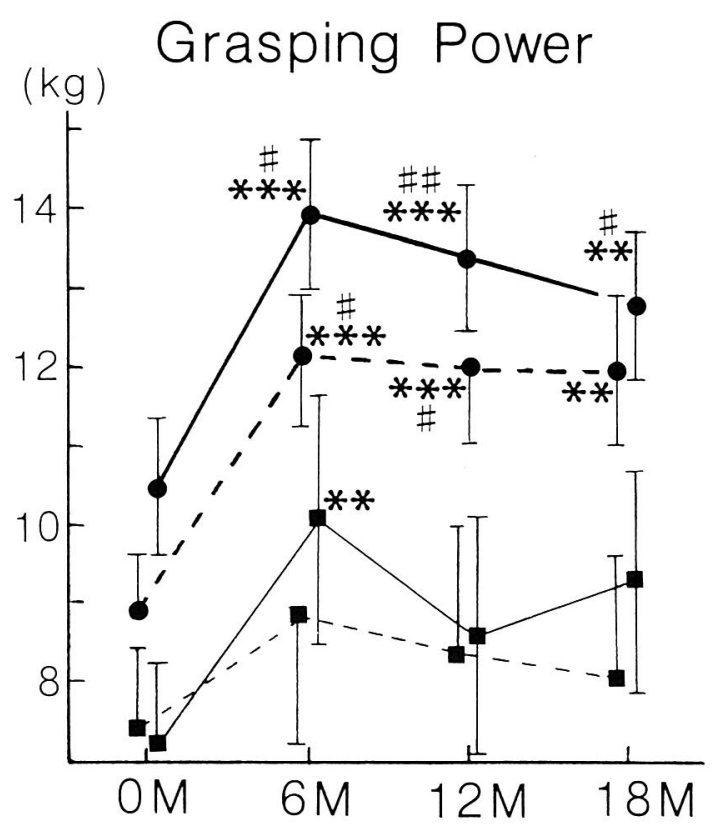

Fig. 2 Changes in grasping power of CT only group $(\boldsymbol{O})$, and CT plus corticosteroid group ( $\mathbf{\square})$. Solid line represents right hand, and broken line, left hand. Values are expressed as the mean \pm SEM. ${ }^{*} p<$ $0.05 ;{ }^{* *} \mathrm{p}<0.01 ;{ }^{* * *} \mathrm{p}<0.001$, comparing before and after the treatment in each group. ${ }^{*} \mathrm{p}<0.05$; $==\mathrm{p}<0.01$, comparison between $\mathrm{CT}$ only and CT plus corticosteroid group. 

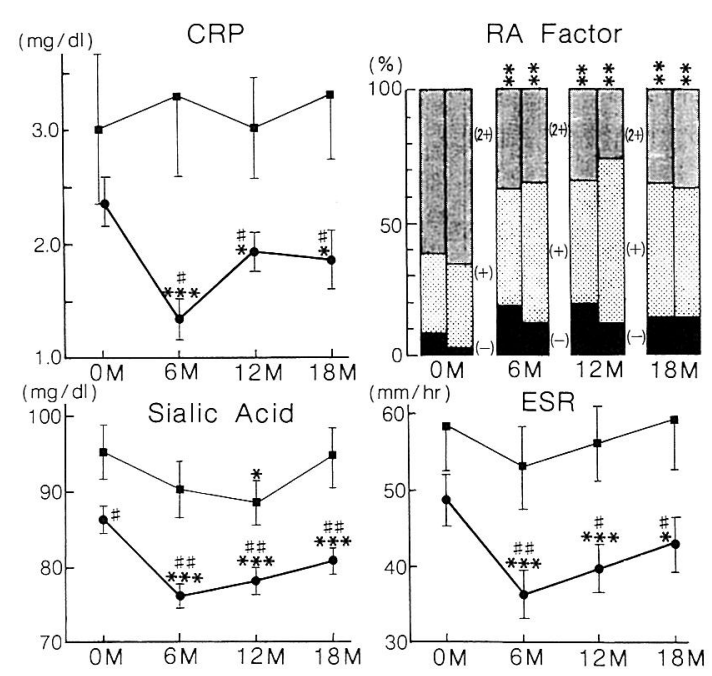

Fig. 3 Changes in CRP, sialic acid, RA factor and ESR of RA patients following the treatments. Symbols and statistics are as in Fig. 2. Left and right bars in RA factor illustrate the CT plus corticosteroid and CT only groups respectively.

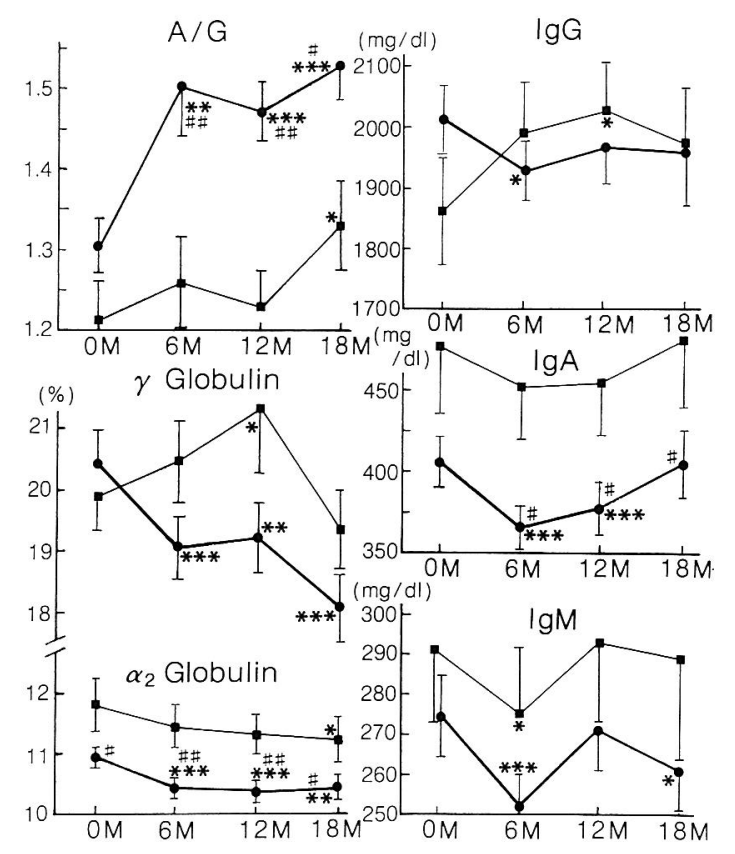

Fig. 4 Changes in A/G ratio, $\alpha_{2}$ globulin, $\gamma$ globulin, $\operatorname{IgG}, \operatorname{IgA}$ and $\mathrm{IgM}$ of RA patients following the treatments. Symbols and statistics are as in Fig. 1.

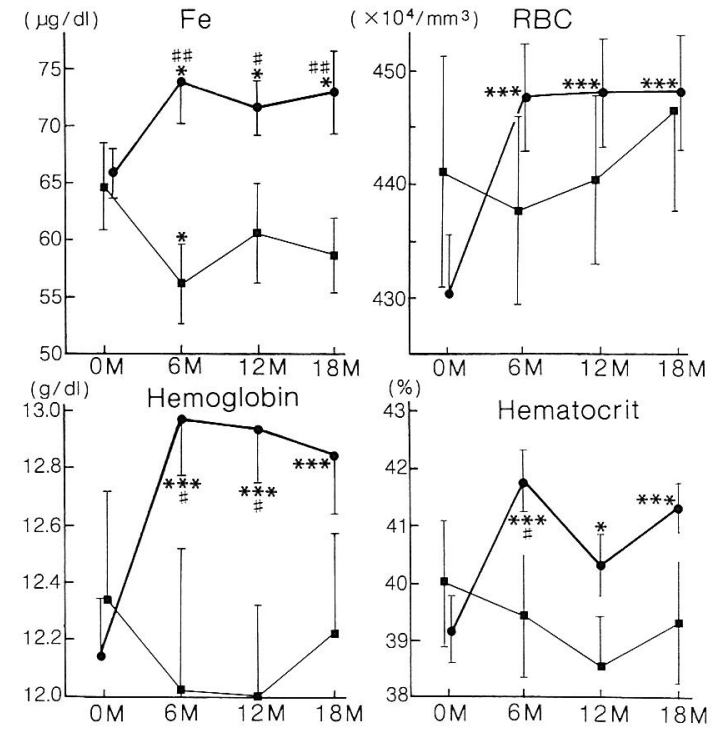

Fig. 5 Changes in serum iron ( $\mathrm{Fe}), \mathrm{RBC}$, hemoglobin and hematocrit of RA patients following the treatments. Symbols and statistics are as in Fig. 1.

different findings were observed. Even before CT treatment, the class and pain score were already heavy, and sialic acid and $\alpha_{2}$ globulin were already elevated as compared with those of the CT only group (Fig. 1, 3, 4). Moreover, in contrast to the CT only group, the levels of $\gamma$ globulin and IgG increased (Fig. 4) and serum iron decreased after CT treatment (Fig. 5). Significant different values were found between two groups after CT treatment in class, pain score, morning stiffness, and grasping power, as well as CRP, sialic acid, ESR, A/G ratio, $\alpha_{2}$ globulin, $\operatorname{Ig} A$, serum iron, hemoglobin, and hematocrit, although some significant improvements were seen in class, pain score, morning stiffness, and grasping power, as well as sialic acid, RA factor, A/G ratio, $\alpha_{2}$ globulin and $\operatorname{IgM}$ (Figs. 1 4).

Nausea and/or flush were each noted in $4 \%$ of the subjects who received CT treatment. Nausea was controlled by the administration of domperidone, $30 \mathrm{mg}$, on the day of CT treatment. The patients experiencing flush found that it was 

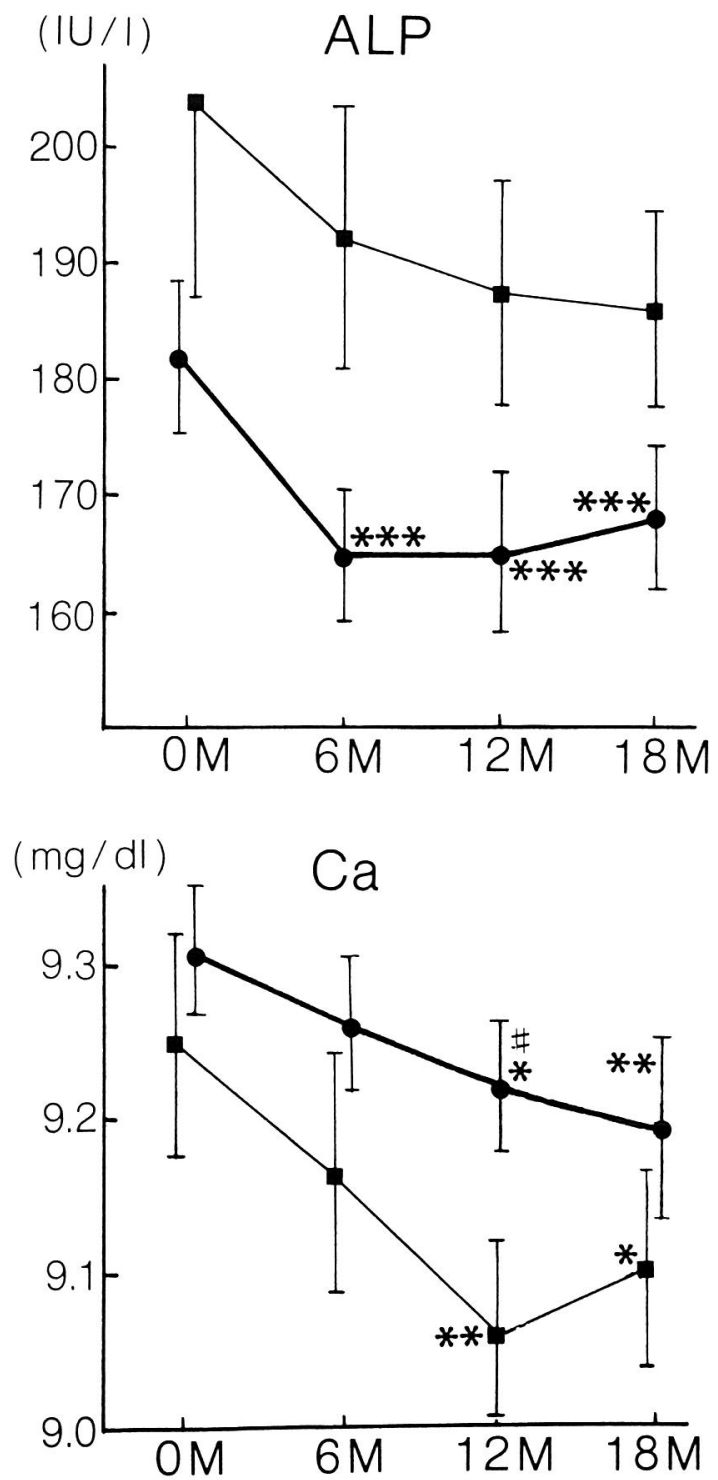

Fig. 6 Changes in ALP and serum calcium ( $\mathrm{Ca}$ ) of RA patients following the treatments. Symbols and statistics are as in Fig. 1.

only temporary and not associated with other signs. No other complications following CT treatment were observed.

\section{Discussion}

The present study has demonstrated that the clinical signs and symptoms of RA as well as various laboratory data were dramatically improved in the $\mathrm{CT}$ only group with comparison to the CT and corticosteroid group. It has been reported that $\mathrm{CT}$ has an anti-inflammatory effect in RA patients ${ }^{1,6)}$. In the CT only group, decreases in CRP, sialic acid and ESR were noted after CT treatment, and these changes would seem to indicate that the inflammatory response was suppressed by $\mathrm{CT}$ (Fig. 3). In addition, reduction of IgG, IgA and IgM levels also occurred and it appeared that these changes resulted in the decreases in $\alpha_{2}$ and $\gamma$ globulins, the elevation of the $A / G$ ratio and the decrease in RA factor (Fig. 4). These results suggest that CT has an effect on the immunological mechanism of RA and the suppression of inflammation was brought about by an immunomodulating effect of $\mathrm{CT}$, since the reduction of immunoglogulins can not be attributed to suppression of the inflammatory response. Therefore, it seems probable that the clinical improvement primarily resulted from the immunomodulating effect of $\mathrm{CT}$, and that suppression of the inflammatory response occurred subsequently. Thus, it appears that CT should be considered as one of the remission inducers for RA.

Our observation suggests that such a small dose of CT, only $20 \mathrm{MRC}$ units/week, does not alter the serum calcium level to such a large quantity (Fig. 6). Kennedy et al. have reported that the bone loss in RA is related to hypercalcemia that follows parathyroid over-activity ${ }^{8}$, and that this state is not associated with a change in the serum calcitonin level ${ }^{9)}$. The calcium level might be mainly regulated by the parathyroid hormone. From the results that CT treatment decreased the serum calcium level even by 0.1 $\mathrm{mg} / \mathrm{d} l$, it is considered that the bone resorption was slightly inhibited by CT treatment (Fig. 6). It is widely know that the serum ALP level is elevated when bone formation is activated ${ }^{11}$. The present study, however, suggested that the activation of bone formation was not brought about 
significantly after CT treatment, because the ALP level fell by CT. Since CT treatment decreased significantly the serum calcium level even with $0.1 \mathrm{mg} / \mathrm{d} l$, the inhibition of bone resorption by CT had partly contributed to the improvement of our RA patients.

The anemia associated with RA is thought to be induced by the deposition of iron in the synovial tissue following repeated small hemorrhages ${ }^{10)}$. From the results that CT improved the anemia, as well as CRP, sialic acid and ESR in the present study (Figs. 3, 5), it is suggested that $\mathrm{CT}$ alleviates the arthritis causing the repeated hemorrhages. Thus, it is assumed that CT prevents further damage to the joints.

Further, corticosteroid treatment appered to interfere with the beneficial effects of CT treatment in terms of clinical signs and laboratory data in RA patients. In the CT plus corticosteroid group, class and morning stiffness were heavy, and sialic acid and $\alpha_{2}$ globulin levels were already high even before CT administration as compared with those in the CT only group (Figs. $1,3,4)$. Thus, it may be considered that both the inflammatory and immunological responses seen in the CT plus corticosteroid group were initially severer than those in the CT only group. However, we did not observe such states of the patients before corticosteroid treatment but after the continuous treatment by the hormones. If the corticosteroid treatment was an effective therapy for RA, the laboratory data in this group would not have been worse than in the CT only group before CT treatment. However, the opposite phenomenon was observed in the present study. Moreover, the interference by this hormone was still recognized even 12 months after the withdrawal of corticosteroid in class, pain score and grasping power, as well as CRP, sialic acid, ESR, $\mathrm{A} / \mathrm{G}$ ratio, $\alpha_{2}$ globulin, $\mathrm{IgA}$, and serum iron (Figs. 1 5). Therefore, it seems possible that the longterm use of corticosteroids hastens the progression of the stage and class in RA.
There is no definitive therapy for RA at present. This study, however, showed that CT treatment produced a significant improvement in RA patients. Therefore, CT can be considered as one of the useful remission inducing drugs for RA. After the reduction of the dose of CT from 20 to 10 MRC units/week, $\operatorname{IgG}, \operatorname{IgA}$ and $\operatorname{IgM}$ levels became insignificant even in the $\mathrm{CT}$ only group (Fig. 3). On the other hand, the serum calcium level was not changed to such a large quantity $(0.1 \mathrm{mg} / \mathrm{d} l)$ in the present study (Fig. 6). From these findings and our preliminary experiences, we like to propose concerning the most adequate dosage of $\mathrm{CT}$ as follows. CT treatment should be started in the dose of 40 MRC units/week by intramuscular administration twice a week until a significant effect is recognized (for $2 \sim 4$ months), and maintained in the dose of $20 \mathrm{MRC}$ units/week by intramuscular administration once a week. When a steady state is obtained, the dose of CT should be increased or decreased by observing the signs and symptoms of each patient. Further, this CT therapy should be started as soon as possible before articular destruction become severer.

\section{References}

1) Abdullahi, S. E., Martelli, E. A., Bramm, E. et al., Effect of calcitonin on different inflammatory models, Agents and Actions, 7 (1977) 533-538.

2) Aida, S., Sato, T. and Simoji, K., The effects of calcitonin on the chronic rheumatoid arthritis, J. New Remedies Clin., 37 (1988) 1203-1207.

3) Aida, S., Tsukui, A. and Simoji, K., The effects of calcitonin on the patient with chronicrheumatoid arthritis, the second report, J. New Remedies Clin., 37 (1988) 1641-1645.

4) Aliapoulis, M. A., Goldhaber, P. and Munson, P. L., Thyrocalcitonin inhibition of bone resorption induced by parathyroid hormone in tissue culture, Science, 151 (1966) 330-331.

5) Arnett, F. C., Edworthy, S. M., Bkoch, D. A. et al., The American Rheumatism Association 1987 Revised criteria for the classification of rheumatoid arthritis, Arthritis. Rheum., 31 
(1988) 315-342.

6) Bobalik, G. R., Aldred, J. P., Kleszynski, R. R. et al., Effects of salmon calcitonin and combination drug therapy on rat adjuvant arthritis, Agents and Actions, 4 (1974) 364-369.

7) Friedman, J. and Reisz, G., Thyrocalcitonin : Inhibitor of bone resorption in tissue culture, Science, 150 (1965) 1465-1467.

8) Kennedy, A. C., Allam, B. F., Boyle, I. T. et al., Abnormalities in mineral metabolism suggestive of parathyroid over-activity in rheumatoid arthritis. Curr. Med. Res. Opin., 3 (1975) 345358.

9) Kennedy, A. C., Allam, B. F., Rooney, P. J. et al., Hypercalcaemia in rheumatoid arthritis, investigation of its causes and implications, Ann. Rheum. Dis., 38 (1979) 401-412.

10) Mowat, A. G. and Hothersall, T. E., Nature of anemia in rheumatoid arthritis, VIII. Iron content of synovial tissue in patients with rheumatoid arthritis and normal individuals, Ann. Rheum. Dis., 27 (1986) 345-351.

11) Ponsen, S., Alkaline phosphatase, Ann. Int. Med., 67 (1967) 183-203.

12) Steinbrocker, O., Traeger, C. H. and Batterman, R. C., Therapeutic criteria in rheumatoid arthritis, JAMA, 140 (1949) 659-662. 